[Supporting Information for:]

Large Scale Fabrication of Aligned Single-Walled Carbon Nanotube Array and Hierarchical Single-Walled Carbon Nanotube Assembly

Junbo $\mathrm{Gao}^{\dagger, \ddagger}$, Aiping $\mathrm{Yu}^{\dagger}$, Mikhail E. Itkis ${ }^{\dagger}$, Elena Bekyarova ${ }^{\dagger}$, Bin Zhao ${ }^{\dagger}$, Sandip Niyogi ${ }^{\dagger}$ and Robert C. Haddon * ${ }^{\dagger}$

${ }^{\dagger}$ Center for Nanoscale Science and Engineering, Departments of Chemistry and Chemical \& Environmental Engineering, University of California, Riverside, California 92521-0403,

${ }^{\ddagger}$ Carbon Solutions, Inc, Riverside, California 92506

\title{
Materials
}

Purified P3-SWNTs were obtained from Carbon Solutions, Inc (www.carbonsolution.com); in order to denote the presence of the carboxylic acid functionality, P3-SWNT material is sometimes referred to as SWNT-COOH. It contains $\sim 3-4 \%$ carboxylic acid groups as estimated by acid-base titration and has a relative carbonaceous purity of $80-$ $90 \%$. Polyvinyl pyrrolidone (PVP, $\left.M_{w} 1,300,000\right)$ and ethanol were purchased from Aldrich.

\section{Solution preparation}

A saturated SWNT suspension was prepared by sonicating SWNTs $(20 \mathrm{mg})$ in a glass vial containing $10 \mathrm{ml}$ of ethanol and $5 \mathrm{ml}$ of DI water. The SWNT suspension was allowed to stand overnight, and the upper layer was decanted and mixed with PVP. The SWNTS/PVP solution was prepared by dissolving PVP $(0.35 \mathrm{~g})$ in $5 \mathrm{ML}$ of a saturated SWNT suspension in ethanol/water ( $v / v, 2: 1)$ with magnetic stirring and the solution was then used for electrospinning experiments. For SWNT diameter measurement before electrospinning, a drop of 20 -folded diluted solution of PVP/SWNTs was placed on a silicon wafer. After evaporation of the solvent at $50{ }^{\circ} \mathrm{C}$, the samples were heated at $600{ }^{\circ} \mathrm{C}$ for 1.5 hour under an argon atmosphere to remove the polymer component and subsequently the AFM observation were carried out. 40 SWNTs were measured to calculate the average diameter before and after the electrospinning.

\section{Fabrication of composite fiber}

The electrospinning experiments were carried out with a high voltage power supply and a glass pipette, with a capillary tip diameter of $1.2 \mathrm{~mm}$. A positive potential was applied by inserting a copper wire into the PVP/SWNT solution in the glass pipette. A single piece of aluminum sheet (one electrode) or two strips of grounded aluminum attached to a polystyrene plate (two electrodes) were used as negative electrodes. The electrospinning experiments were carried out at a voltage of $5 \mathrm{KV}$ with the glass pipette held $5 \mathrm{~cm}$ from the negative electrode.

\section{Polymer removal by chemical vapor etch method}

To remove PVP from the electrospun fiber by chemical vapor, the composite fibers were first deposited on a silicon wafer. Then this wafer was placed horizontally in a $50 \mathrm{ml}$ beaker containing $20 \mathrm{ml}$ ethanol with a $0.2-0.5 \mathrm{~cm}$ distance above the methanol surface. Subsequently the beaker was sealed by aluminum foil. 
Figure 1 (enlarged for clarity).
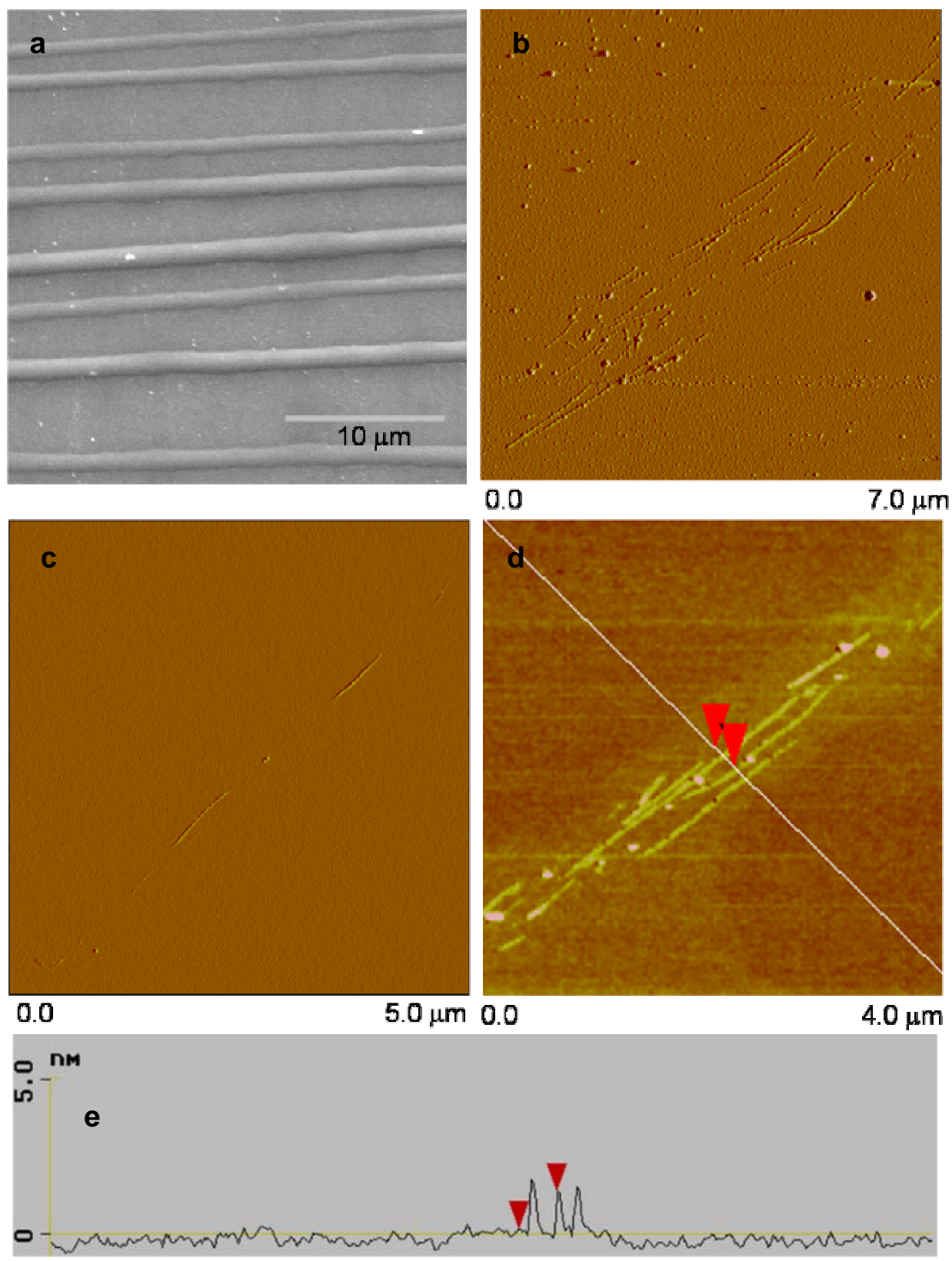

Figure 1. (a) SEM image of aligned electrospun fibers. (b-d) AFM images of aligned SWNTs prepared by heating the electrospun fiber: (b) aligned SWNT array, (c) single line of SWNTs, (d) aligned individual SWNTs, and (e) their height cross-sectional analysis. 
Figure 2 (enlarged for clarity).

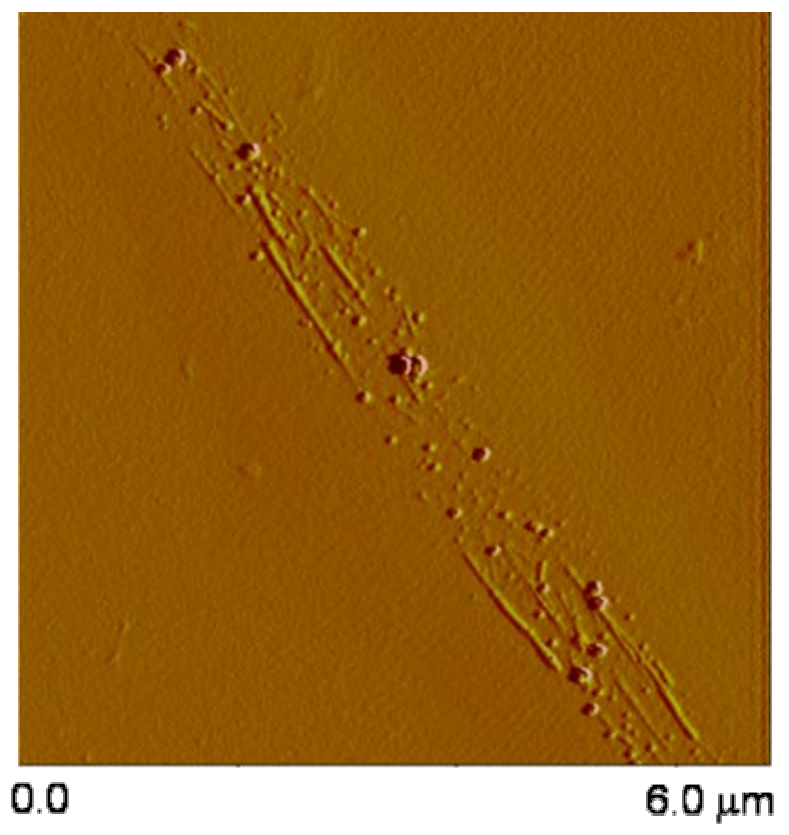

Figure 2. AFM image of aligned SWNTs prepared by ethanol vapor etching method. 
Figure 3 (enlarged for clarity).

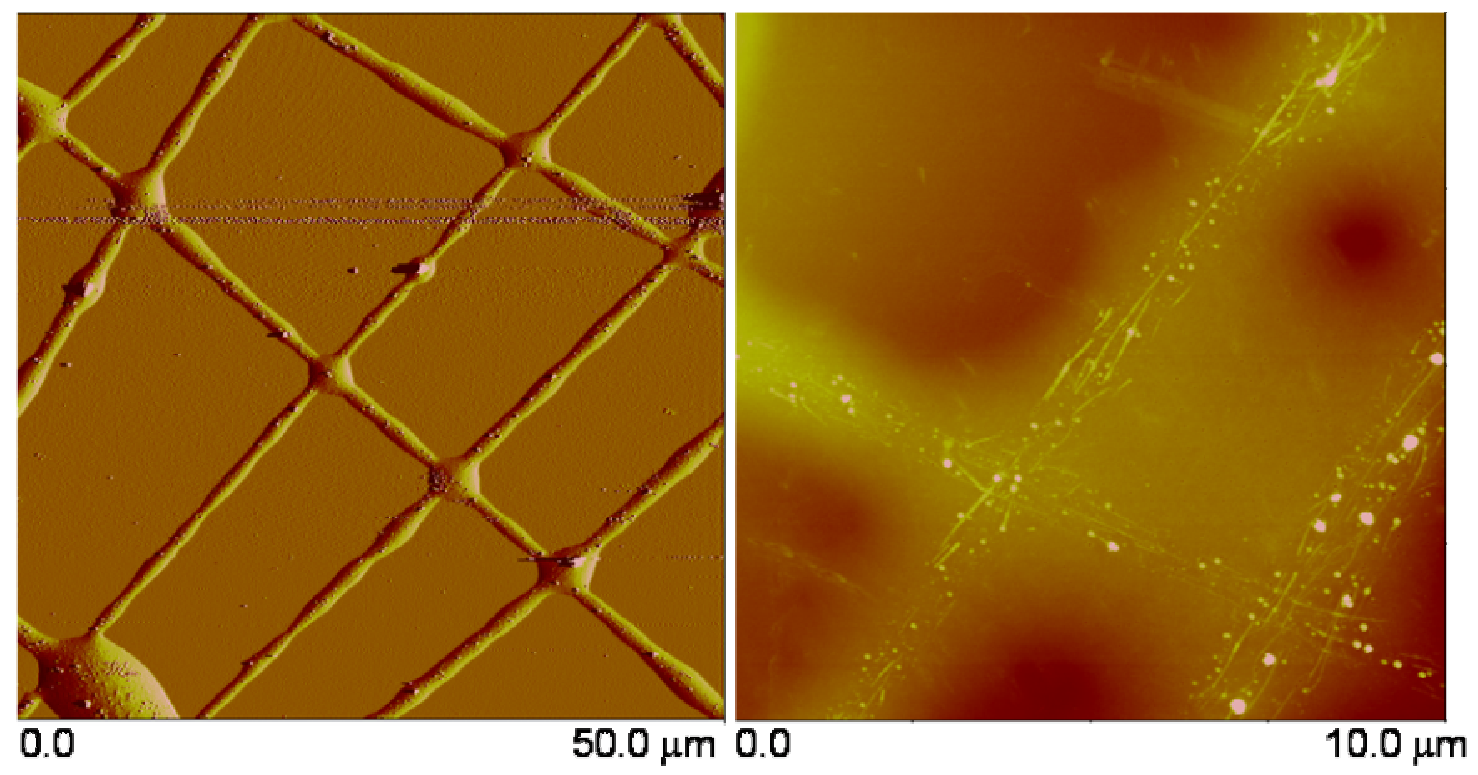

Figure 3. AFM images of (a) cross-aligned SWNTs/PVP composite fibers, and (b) cross aligned SWNT array. 


\section{Supporting Figures}

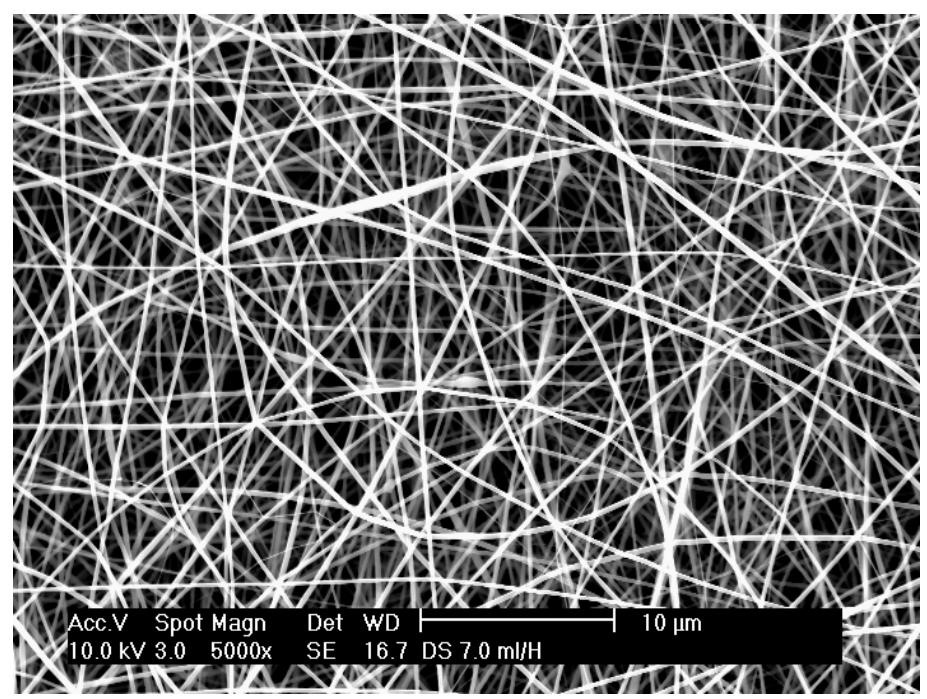

Supporting Figure 1. SEM images of SWNTS/PVP fiber prepared using one negative electrode 


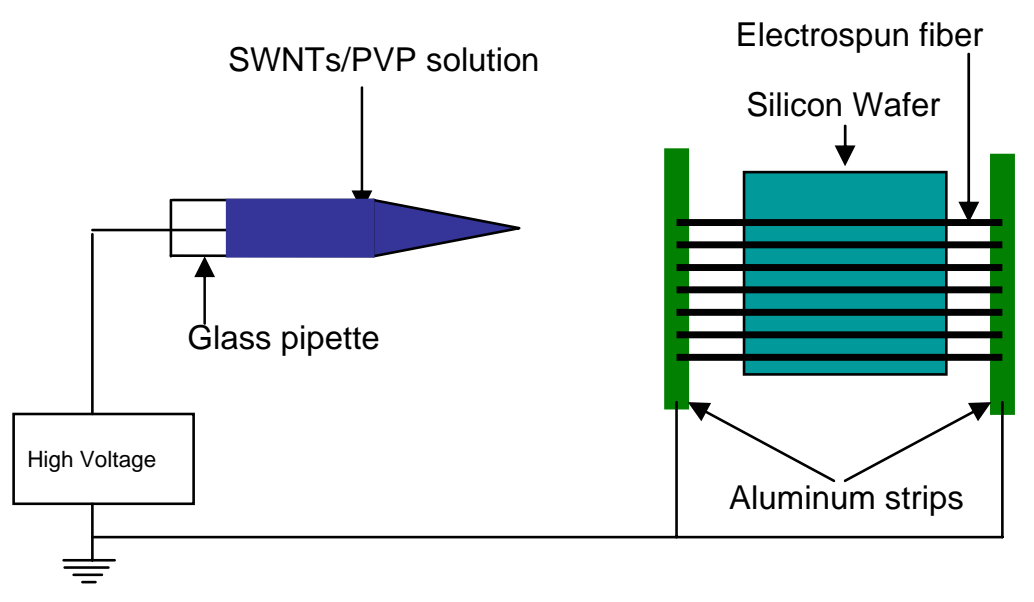

Supporting Figure 2. Schematic of electrospinning set-up using two pieces of aluminum strip as negative electrode. 


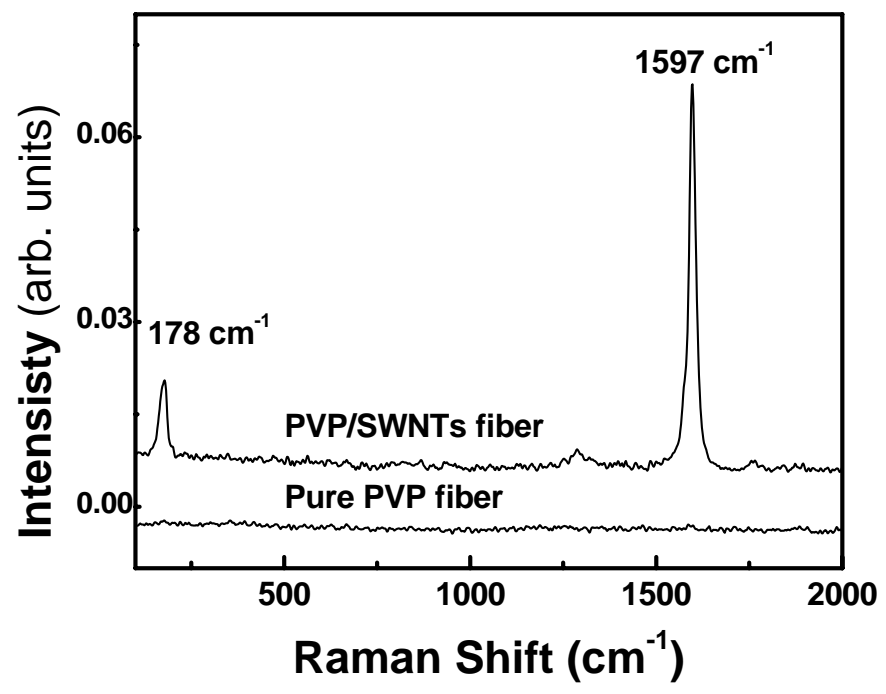

Supporting Figure 3. Raman spectra of pure PVP fiber and SWNTs/PVP composite fiber 